\title{
Welcome to Dresden
}

Die Universitätshautklinik heißt alle Teilnehmer der 43. DDG-Tagung vom 20.-23. 4. 2005 in Dresden herzlich willkommen.

Die Dermatologen in Dresden und im gesamten sächsischen Raum sind stolz darauf, dass Dresden in diesem Jahr zum zweiten Mal nach 1925 wieder Austragungsort einer Jahrestagung der Deutschen Dermatologischen Gesellschaft ist. Als Direktor der Klinik und Poliklinik für Dermatologie am Universitätsklinikum Carl Gustav Carus der Technischen Universität Dresden habe ich mich sehr gefreut, von der Tagungsleitung in das Programmkomitee berufen worden zu sein. Ich darf die Gelegenheit nutzen, den Lesern der „Aktuellen Dermatologie“ kurz unsere Klinik vorzustellen. Wir sind noch eine relativ junge Einrichtung, die 1957 aus der Poliklinik des ehemaligen Stadtkrankenhauses DresdenJohannstadt entstanden ist. Diese erste Dresdner Hochschulhautklinik gehörte zu der Medizinischen Akademie Dresden (MAD), die bereits 1954 gegründet worden war. Erster Direktor der Hochschulhautklinik war Prof. H.-E. Kleine-Natrop, der zuvor als leitender Oberarzt der Universitätshautklinik Kiel Erfahrungen zum Aufbau einer Klinik sammeln konnte. Dank der internationalen Verbindungen und des diplomatischen Geschicks von H.-E. Kleine-Natrop wurde die Dresdner Hochschulhautklinik, trotz der bekannten DDR-Restriktionen eine angesehene Institution. Nach der Wende wurde die ehemalige MAD in die Medizinische Fakultät Carl Gustav Carus der Technischen Universität überführt und unsere Klinik erhielt als Universitätshautklinik nun eine gesicherte Perspektive mit allen Möglichkeiten der modernen Dermatologie in der Krankenversorgung, der Lehre und Forschung. Nach dem Direktorat von Prof. J. Barth übernahm Prof. G. Richter im Januar 1985 die kommissarische Leitung der Klinik und der Poliklinik für Hautkrankheiten. Prof. Richter, Inhaber der Schwerpunktprofessur „Allergologie und Berufsdermatologie“ hat nicht nur auf seinem Fachgebiet national und interna- tional anerkannte Arbeit geleistet, sondern auch die Klinik umsichtig in die akademische Zukunft geleitet. Unterstützt wurde er dadurch vor allem von Prof. Gottfried Wozel, der maßgeblich in der Gründungskommission für die neue Medizinische Fakultät an der TU Dresden mitgewirkt hatte und auf die Schwerpunktprofessur „Dermatopharmakologie“ an unserer Klinik berufen wurde, wie von vielen anderen Kollegen, die mit Elan und Optimismus das Leistungsspektrum unserer Klinik erweitern konnten. Es war für mich eine große Ehre, Anfang 1997 die Leitung der Klinik und Poliklinik für Dermatologie am Universitätsklinikum Carl Gustav Carus Dresden der TU Dresden zu übernehmen. Die Dresdner Dermatologie war stets von einer kollegialen $\mathrm{Zu}$ sammenarbeit geprägt, nicht nur mit den niedergelassenen Dermatologen der Stadt und der Region, sondern vor allem auch mit der städtischen Hautklinik Friedrichstadt, die damals von Prof. Claus Seebacher geleitet wurde. Wichtiger Stützpunkt für diese kollegiale Zusammenarbeit ist unsere traditionelle starke Poliklinik, die bis 1996 von Prof. A. Scholz geleitet wurde, der später auf den Lehrstuhl der Geschichte für Medizin unserer Fakultät berufen wurde. Die behutsame Überführung der allgemeinen dermatologischen Hautpoliklinik in einen integrierten Verbund von Spezialambulanzen hat für diese Einrichtung ein stetiges und gesundes Wachstum auch in den Zeiten der Gesundheitsreform ermöglicht. $\mathrm{Zu}$ nennen sind hier die hervorragend ausgestattete und damit größte Lasereinrichtung in Ostdeutschland, die von Dr. Ingrid Hackert und OA Roland Aschoff geleitet wird; eine sehr aktive phlebologische Ambulanz unter der Leitung von OÄ Ulrike Köhler, die Spezialsprechstunden für Autoimmunkrankheiten, für Kollagenosen und Blasenbildende Autoimmunkrankheiten (OÄ Christiane Pfeiffer) und die Immunschwächeambulanz, die sich unter der engagierten Leitung von OÄ Petra Spornraft-Ragaller zur zentralen interdisziplinären ambulanten Versorgungseinrichtung für HIV-infizierte Menschen in Ostsachsen 
entwickelt hat. Einen besonderen Aufschwung im ambulanten Bereich hatte der Bereich Allergologie genommen, der nach der Emeritierung von Prof. Richter durch OÄ Petra Spornraft-Ragaller und OA Knut Schäkel geleitet wird. Erfolgreich war u.a. hier die Einrichtung einer Neurodermitisspezialsprechstunde und die Etablierung der Eltern-Patienten-Schulung für Neurodermitiskranke. Im stationären Bereich der dermatologischen Universitätsklinik Dresden, der in einen konservativen und onkologischen Teil gegliedert ist, kam es in den letzten Jahren zu deutlichen Strukturveränderungen, die einerseits zu einer Betten- und Personalreduktion, andererseits aber auch zu einer Steigerung der Fachabteilungsfälle auf über 2000 Patienten jährlich bei deutlich reduzierter Verweildauer auf durchschnittlich 5,6 Tage geführt hat. Einen wesentlichen Anteil an dieser sehr positiven Entwicklung hat auch der operativ-onkologische Bereich unserer Klinik, der unter der langjährig bewährten Leitung von Prof. Günther Sebastian und OÄ Annette Stein steht. Dieser Bereich zeichnet sich nicht nur durch die hervorragende operative Versorgung der Patienten, sondern auch durch die innovative und interdisziplinäre, stationäre und in der Nachsorge ambulante Versorgung unserer onkologischen Patientinnen und Patienten aus. Auch der konservativ-stationäre Teil der Klinik hat vor allem durch die Einführung moderner Therapieverfahren in der Behandlung von Psoriasis, schweren Ekzemformen und Autoimmunerkrankungen einen großen Aufschwung erfahren. Im Bereich Forschung stehen die Ampeln in unserer Klinik auf grün. Genannt seien das Vitamin $\mathrm{D}_{3}$ Labor von Dr. Bodo Lehmann, das photobiologische Labor von Dipl.phys. Peter Knuschke, der Bereich Autoimmundiagnostik von OÄ Christiane Pfeiffer, die immunologische Forschung (OA Knut Schäkel und Dr. Claudia Günther), das Histologielabor (OÄ Annette Stein und Dr. Jörg Laske) sowie der Bereich innovative Therapie und Diagnostik unter der Leitung von OA Roland Aschoff. Durch die Allianz der Medizi- nischen Fakultät Carl Gustav Carus Dresden mit der Havard Medical School in Boston/USA haben wir die Möglichkeit erhalten, den gesamten akademischen Unterricht nach den Prinzipien des Problem-/Patientenorientierten Lernens zu reformieren. Groß geschrieben wird an unserer Universitätshautklinik auch der Bereich Fort- und Weiterbildung. Hier führen wir die bereits 1959 von H.-E. Kleine-Natrop gegründeten „Dresdner Dermatologischen Gespräche“ mit großer Resonanz fort; zum festen Programm im Jahresablauf gehören auch die Interdisziplinären Symposien für Allergologie, die stets gut besuchten Aids-Kolloquien, die von Prof. Günther Sebastian gegründeten und geleiteten Melanom-Workshops sowie zahlreiche Veranstaltungen mit Referenten aus Dresden, Deutschland und der ganzen Welt. Unsere Gastgeberrolle wird durch die Attraktivität von Dresden sehr erleichtert. In diesem Sinne hoffen alle Mitarbeiterinnen und Mitarbeiter der Klinik und Poliklinik für Dermatologie am Universitätsklinikum Carl Gustav Carus der Technischen Universität Dresden, dass die Gäste, die anlässlich der 43. Tagung der Deutschen Dermatologischen Gesellschaft in Dresden weilen, auch Gelegenheit finden, die Schönheiten unserer einmaligen Stadt und Kulturlandschaft auf sich wirken zu lassen.

\section{Prof. Michael Meurer}

Direktor der Klinik

\section{Literatur}

${ }^{1}$ Scholz A, Roder H. Ein Stück Geschichte der Dermatologie in Dresden. NTM-Schriftenreihe. 1973; 10: 68 - 74

${ }^{2}$ Hansel G. 120 Jahre Hautklinik Dresden-Friedrichstadt. Der Hautarzt 1995; 46: $278-282$

${ }^{3}$ Richter G. 40 Jahre Hautklinik Dresden. Von der Akademie zur Universität. Der Hautarzt 1997; 48: 671-673 